\title{
Long-term feeding effect of mono and divalent cation Kappa Carrageenan on some antioxidant, biochemical and histological parameters of growing male rats
}

\author{
A. Farag ${ }^{1}$, N.M. Zahran ${ }^{2}$, A.S. Salem ${ }^{3}$ and H.A. Awney ${ }^{4}$ \\ ${ }^{1}$ Department of Environmental Studies, Institute of Graduate Studies and Research, Alexandria University, Egypt, \\ ${ }^{2}$ Department of Histopathology, Faculty of Medicine, Alexandria University, Egypt, ${ }^{3}$ Animal Production Research \\ Institute, Agriculture Research Center, Cairo, Egypt and ${ }^{4}$ Department of Environmental Studies, Institute of Graduate \\ Studies and Research, Alexandria University, Egypt.
}

Kappa carrageenan $(\mathcal{K} \mathrm{CGN})$ is non-caloric, non-nutritive food additive (E407) widely used for their gelling properties in low fat food and infant formula. The gel formation of $k \mathrm{CGN}$ depends on the type and concentration of certain cations such as $\mathrm{Na}$ and $\mathrm{Ca}$. Previous study in our lab indicated that gel formation of $k$ CGN with $10 \% \mathrm{Na}^{+}$or $10 \% \mathrm{Ca}^{++}$exhibits optimal gelling pattern. Despite the GRAS status of $k$ CGN, its safety for human consumption has long been questioned. Due to its high molecular weight and strong interacting with proteins, $k$ CGN slow the enzymatic digestion of protein, affects protein bio-accessibility, bioavailability and overall physiological consequences ${ }^{(1,2)}$. In 2015, JECFA noted that no studies were available addressing the effects of $k$ CGN on the immature gut of neonatal ${ }^{(3)}$. This study aimed to examine the effects of $\mathrm{Na}^{+}$and $\mathrm{Ca}^{++}$dependent $k$ CGN gel on serum biochemical parameters, serum and liver antioxidant levels and colon histological features of neonatal male rats. The local committee approved the design of the experiment and the protocol conforms to the guidelines of the National Institute of Health (NIH).

Four groups of three-week-old male Sprague-Dawley rats received daily oral dose of pure refined food grade $k \mathrm{CGN}$ (500 mg $/ \mathrm{kg}$ $\mathrm{BW} /$ day), $\mathcal{K} \mathrm{CGN}$ with $\mathrm{Na}\left(500 \mathrm{mg} / \mathrm{kg} \mathrm{BW} /\right.$ day $\left.+10 \% \mathrm{Na}^{+}\right), k \mathrm{CGN}$ with $\mathrm{Ca}\left(500 \mathrm{mg} / \mathrm{kg} \mathrm{BW} / \mathrm{day}+10 \% \mathrm{Ca}{ }^{++}\right)$or no $k \mathrm{CGN}$ as control group for 14 weeks. All doses were prepared daily based on the most recent body weight measurements. During the study, all groups showed normal growth without significant difference in body weight or feed consumption rate. No mortality or pathological symptoms were observed.

Serum biochemical parameters showed significant increase $(\mathrm{P}<0.05)$ in total cholesterol for rats treated with $k \mathrm{CGN}+\mathrm{Ca}(86.20 \pm$ $2.58 \mathrm{mg} / \mathrm{dl})$ compared with control $(74.60 \pm 6.80 \mathrm{mg} / \mathrm{dl})$. However, no significant differences have been observed in total glycerides, total lipids, high density lipoprotein cholesterol (HDL), low density lipoprotein cholesterol (LDL), liver enzymes, urea, creatinine or hematological parameters between all groups. Serum antioxidant biomarkers in rats treated with $k \mathrm{CGN}+\mathrm{Ca}$ showed significant decreased $(\mathrm{P}<0.05)$ in both superoxide dismutase (SOD) activity $(156.92 \pm 9.34 \mathrm{U} / \mathrm{ml})$ compared with control $(209.60 \pm 32.59 \mathrm{U} / \mathrm{ml})$ and glutathione reductase $(\mathrm{GR})$ activity $(3.23 \pm 0.16 \mathrm{U} / \mathrm{ml})$ compared with control $(7.39 \pm 0.38 \mathrm{U} / \mathrm{ml})$. No significant difference was detected in serum SOD or GR between $k \mathrm{CGN}, k \mathrm{CGN}+\mathrm{Na}$ and control. Rats treated with $k$ CGN showed significant decrease $(\mathrm{P}<$ $0.05)$ in malondiadehyd (MDA) level $(0.47 \pm 0.16 \mathrm{nmol} / \mathrm{ml})$ compared with control $(0.84 \pm 0.14 \mathrm{nmol} / \mathrm{ml})$ whereas no significant different was detected between $k \mathrm{CGN}+\mathrm{Na}, k \mathrm{CGN}+\mathrm{Ca}$ and control. Catalase activity (CA) exhibits no significant difference between all groups. On the other hand, liver antioxidant parameters showed no significant differences in SOD, GR, MDA or CA levels between all groups. Histological investigation showed minor changes in rat colon mucosa of $k \mathrm{CGN}$ and $k \mathrm{CGN}+\mathrm{Na}$ such as focal destruction of crypt of lieberkuhn where some are replaced by cellular infiltration, loss of colonic crypt integrity as loss of vacuolated cytoplasm, presence of dense small nuclei and lymphocytic aggregation in the submucosa. Anatomical examination of GIT indicated severe gathering of gases in colon of rats treated by $k$ CGN only.

In a conclusion, gel formation of $k$ CGN with $10 \% \mathrm{Na}^{+}$or $10 \% \mathrm{Ca}^{++}$exhibits more strength in gelling pattern which may reduce the amount of CGN needed to obtain optimal gel properties. However, significant changes in some antioxidant, biochemical and histological parameters in the growing male rats may limit the practicality of these findings. The present study appears useful in leading to further investigations concerning the effects of $k$ CGN on neonatal GIT.

1. Weiner ML. 2014. Crit Rev Toxicol. 44(3):244-69.

2. Weiner ML, Ferguson HE, Thorsrud BA et al., 2015. Food Chem Toxicol. 77: 120-131.

3. Joint FAO/WHO Expert Committee on Food Additives (JECFA) WHO food additive series: 70, 2015. 